\title{
The Inviscid Text Entry Rate and its Application as a Grand Goal for Mobile Text Entry
}

\author{
Per Ola Kristensson \\ University of St Andrews, UK \\ pok@st-andrews.ac.uk
}

\author{
Keith Vertanen \\ Montana Tech, USA \\ kvertanen@mtech.edu
}

\begin{abstract}
We introduce the concept of the inviscid text entry rate: the point when the user's creativity is the bottleneck rather than the text entry method. We then apply the inviscid text entry rate to define a grand goal for mobile text entry. Via a proxy measure we estimate the population mean of the sufficiently inviscid entry rate to be $67 \mathrm{wpm}$. We then compare existing mobile text entry methods against this estimate and find that the vast majority of text entry methods in the literature are substantially slower. This analysis suggests the mobile text entry field needs to focus on methods that can viably approach the inviscid entry rate.
\end{abstract}

\section{Author Keywords}

Inviscid entry rate; mobile text entry; crowdsourcing

\section{ACM Classification Keywords}

H.5.2 Information interfaces and presentation: User Interfaces: Input devices and strategies

\section{INTRODUCTION}

It is obvious text entry methods should be fast. However, what is not obvious is how we know when we have created a text entry method that is fast enough. The maximum upper bound for text entry is the speed of light, which is the fundamental limit for how fast physical information can travel. We also note that users can speak very fast, up to $200 \mathrm{wpm}$ [16]. However, can users compose non-trivial text at such a high entry rate? The processes of speaking and writing, while related, are significantly different (see Chafe and Tannen [2] for an extensive overview). To put this further into perspective, an A4 sheet of paper contains about 500 words. To be able to write an email at 200 wpm means being able to write a short essay filling an entire A4 sheet in just 2.5 minutes.

We introduce the concept of the inviscid entry rate. We argue the search for faster text entry methods is bounded: there is a limit when it is not useful to search for faster text entry methods. This point occurs when the text entry method is not the bottleneck of the process of transferring thoughts into writing. In other words, the point when the user's creativity is the bottleneck rather than the text entry method. We call this

\footnotetext{
Permission to make digital or hard copies of all or part of this work for personal or classroom use is granted without fee provided that copies are not made or distributed for profit or commercial advantage and that copies bear this notice and the full citation on the first page. Copyrights for components of this work owned by others than ACM must be honored. Abstracting with credit is permitted. To copy otherwise, or republish, to post on servers or to redistribute to lists, requires prior specific permission and/or a fee. Request permissions from permissions@ acm.org.

MobileHCI 2014, September 23-26, 2014, Toronto, ON, Canada.

Copyright (C) 2014 ACM 978-1-4503-3004-6/14/09 ...\$15.00.

http://dx.doi.org/10.1145/2628363.2628405
}

point the inviscid entry rate. The term inviscid is borrowed from fluid dynamics: an inviscid fluid has no viscosity. Similarly, an inviscid text entry method does not hinder the free flow of information from a user's mind to the computer.

The inviscid entry rate can be estimated by measuring how fast users can compose novel messages using an inviscid text entry method. An entry rate depends on the amount of text generated and the time it takes to write it. Let us define $T_{\text {compose }}$ as the time it takes to a compose a given amount of text and $T_{\text {transcribe }}$ as the time to transcribe the same amount of text. $T_{\text {compose }}$ can be broken down into two components- the time it takes to conceive the text ( $\left.T_{\text {conceive }}\right)$, and the time it takes to articulate the text $\left(T_{\text {articulate }}\right)^{1}$ :

$$
T_{\text {compose }}=T_{\text {conceive }}+T_{\text {articulate }} \text {. }
$$

In contrast, the time it takes to transcribe memorable ${ }^{2}$ text depends solely on the time it takes to articulate the text:

$$
T_{\text {transcribe }}=T_{\text {articulate }} \text {. }
$$

For a given fixed amount of generated text, an inviscid text entry method will result in $T_{\text {compose }}$ being markedly higher than $T_{\text {transcribe }}$. This is because when the text entry method is inviscid, it is rate-limited by $T_{\text {conceive }}$. At this point, even if $T_{\text {articulate }}$ is further reduced, it will only result in a negligible performance improvement.

We currently do not know if a true inviscid text entry method has been discovered. However, we can still identify a sufficiently inviscid text entry method. A sufficiently inviscid text entry method is a text entry method in which $T_{\text {compose }}$ is markedly higher than $T_{\text {transcribe }}$.

We demonstrate how to apply the concept of the inviscid entry rate to form a meaningful grand goal for mobile text entry. If we have a population estimate of users writing with a sufficiently inviscid text entry method, then we have an explicit goal to aspire to in the field of mobile text entry. The creative process of inventing a new mobile text entry method is then not about creating faster text entry methods in comparison to what is out there. Instead, it is about creating mobile text entry methods performing at, or slightly above, the sufficiently inviscid entry rate. After that point, it could be more useful to address other design aspects of text entry (such as for instance user engagement or learnability).

\footnotetext{
${ }^{1}$ Articulation time includes not only motor actions but also fixed overhead time spent processing visual feedback.

${ }^{2}$ The established experimental paradigm for testing text entry methods is to instruct participants to transcribe memorable sentences.
} 
We hypothesize that we can estimate a sufficiently inviscid entry rate for mobile text entry by measuring how fast users compose small messages on their regular full-sized QWERTY keyboards. The assumption is that the regular fullsized QWERTY keyboard is a sufficiently inviscid text entry method. This is plausible for two reasons. First, the fact that the ubiquitous full-sized QWERTY keyboard has prevailed means that it is likely that most users are satisfied with its speed [4]. Second, as we will show, users compose text significantly slower than they transcribe text when they use their regular full-sized QWERTY keyboards.

This means we can measure the text entry rate from a wide sample of users composing novel messages using their own full-sized keyboards and infer a sufficiently inviscid entry rate. Assuming a normal distribution, by obtaining a population estimate of the mean composition entry rate and adding one standard deviation we acquire a sufficiently inviscid entry rate that represents a text entry rate about $84 \%$ of users in the population cannot surpass if they compose original text using a full-sized desktop or laptop keyboard.

As a control, we also measure participants' transcription entry rate. If the mean composition entry rate is markedly lower than the mean transcription entry rate then the bottleneck for composition was not the text entry method per se but the creativity required to invent novel messages.

\section{ESTIMATING A SUFFICIENTLY INVISCID ENTRY RATE}

We used data originally collected as part of Experiment 1 in Vertanen and Kristensson [20]. In this paper, we re-analyzed the data using only workers resident in the US. This was done to focus on fluent speakers who are likely faster typists. It also eliminated some non-US workers who appeared to be copying text rather than creating novel compositions. Removing non-US workers reduced the number of participants from 181 to 115 . We additionally removed one composition task that was not representative of mobile text entry (the AID condition in [20]). For completeness, we explain the methodology of the original data collection.

We recruited 115 participants from the US using Amazon's Mechanical Turk microtask market. They were compensated \$1 for taking part in a single Human Intelligence Task (HIT). The average time to complete the HIT was 27 minutes. Before the first task, we asked participants to provide information such as sex, country, age, English ability, typing ability, and computer type.

Participants were asked to complete four tasks using their full-sized keyboards. In each task they did ten different transcriptions or compositions. The order of the tasks was randomized. In all the composition tasks participants were instructed not to disclose any private information (such as phone numbers, email addresses or names). Participants were prevented from pasting text. For the exact instructions given for each task, see Figure 1 in [20]. The four task types were:

Transcription Task - Participants were presented with a sentence and asked to type it as quickly and as accurately as possible. The sentences were taken from a corpus of email messages written by Enron employees on their BlackBerry mobile devices [19]. This was the COPY condition in [20].

Composition Task 1 - Participants were presented with a short message (e.g. "Wanna go to the park?") and asked to imagine they received this message on a mobile device. They were asked to type a fictitious response. The set of messages used in this task were created by other workers who had been asked to invent things they might send from a mobile device. This was the REPLY condition in [20].

Composition Task 2 - Participants were presented with short fictitious situations (e.g. "Your co-worker Carl will be 29 years old today. Send him a greeting."). They were then asked to imagine they were using a mobile device to compose a response to this situation. This was the SITUATION condition in [20].

Composition Task 3 - Participants were asked to imagine they were using a mobile device and to compose a fictitious message. They were also advised that if they were struggling for ideas to think about things they write on their own mobile device. This was the COMPOSE condition in [20].

\section{Results}

The average age of participants (self-reported) was 29 (sd = 10). $64 \%$ reported being female. Participants rated their English skill as native $(96 \%)$ or advanced $(4 \%)$. Participants reported using a laptop $(62 \%)$ or a desktop computer (38\%). Participants reported their typing ability as fast (37\%), moderate $(59 \%)$ or slow $(3 \%)$.

For the transcription task, error rate was measured as the minimum edit distance between the stimulus sentence and the participant's response, divided by the number of characters in the stimulus sentence. This provides us with a characterlevel error rate. We found that the mean error rate was $2.2 \%$ and the median error rate was $0 \%$. This is comparable with other text entry experiments (e.g. [14]).

Participants' entry rate was measured in words-per-minute (wpm) and a word was defined as five consecutive characters including spaces. The time required to write a response was defined as the interval between when they first typed a character in response to a new stimulus until their last key press before proceeding to the next stimulus. This meant that if participants paused between stimuli, such pauses did not affect the entry rate measurements. The mean entry rate for the transcription task was $68 \mathrm{wpm}$. Participants transcribed a little over $11 \mathrm{~K}$ words.

For the composition tasks, we verified participants' responses were intelligible and sensible, removing from our analysis any participants who did not provide adequate responses. Overall participants created plausible and imaginative compositions (for examples see [20]). Entry rate was measured in the same way as for the transcription task. In total, participants composed a little over $29 \mathrm{~K}$ words.

Figure 1 shows the entry rate for each task. Participants wrote at $57 \mathrm{wpm}(\mathrm{sd}=20)$ in composition task $1,43 \mathrm{wpm}(\mathrm{sd}=13)$ in composition task 2 , and $55 \mathrm{wpm}(\mathrm{sd}=18)$ in composition 


\begin{tabular}{ll}
\hline Text entry method & $\begin{array}{l}\text { Highest reported } \\
\text { entry rate (wpm) }\end{array}$ \\
\hline Estimate of the inviscid entry rate & 67 \\
\hline Physical thumb keyboards & $60[3]$ \\
Gesture keyboards & $45[9]$ \\
Optimized on-screen keyboards & $45[12]$ \\
QWERTY on-screen keyboards & $40[12]$ \\
KALQ thumb keyboard & $37[14]$ \\
Half-QWERTY & $35[13]$ \\
Twiddler & $35[11]$ \\
WalkType & $31[5]$ \\
ContextType & $28[6]$ \\
Disambiguating keypads & $26[7]$ \\
Unconstrained handwriting recognition & $25[8]$ \\
Dasher & $20[21]$ \\
Mobile speech & $18[18]$ \\
Quikwriting & $16[15]$ \\
Unistrokes & $16[1]$ \\
TiltText & $14[22]$ \\
Multi-tap & $12[23]$ \\
Graffiti & $11[1]$ \\
EdgeWrite & $7[24]$ \\
\hline
\end{tabular}

Table 1. The highest reported entry rate in words-per-minute (wpm) of a variety of mobile text entry interfaces.

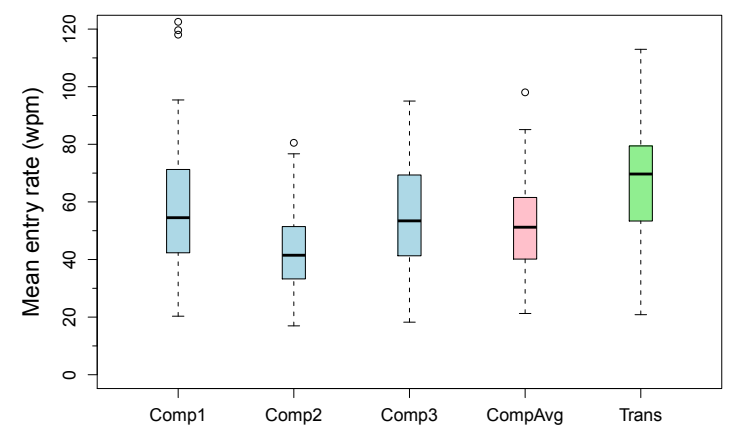

Figure 1. Entry rates for the composition and transcription tasks.

task 3 . The grand mean composition entry rate was $52 \mathrm{wpm}$ $(\mathrm{sd}=15)$. For comparison, the transcription entry rate was 68 wpm $(s d=18)$. According to our previous definition of the inviscid entry rate being equal to a population estimate of the mean composition entry rate plus one standard deviation, the inviscid entry rate in the population is $67 \mathrm{wpm}$.

Figure 2 shows the entry rate distribution for the transcription task and the three composition tasks. As shown in Figure 2, all composition tasks follow a roughly similar positively skewed distribution with a mode relatively close to each other. Thus, to estimate the inviscid entry rate we averaged the mean composition rates in Figure 1.

The transcription entry rate (68 wpm) was markedly higher than the mean composition entry rate (52 wpm). Thus, it is likely the cognitive process of composing text $\left(T_{\text {conceive }}\right)$ was the bottleneck rather than the operation of a full-sized keyboard $\left(T_{\text {articulate }}\right)$. RM-ANOVA revealed that the difference between mean composition entry rate (averaged over all three composition tasks) and the transcription rate was significant $\left(F_{1,114}=125.969, \eta_{p}^{2}=0.525, p<0.001\right)$.

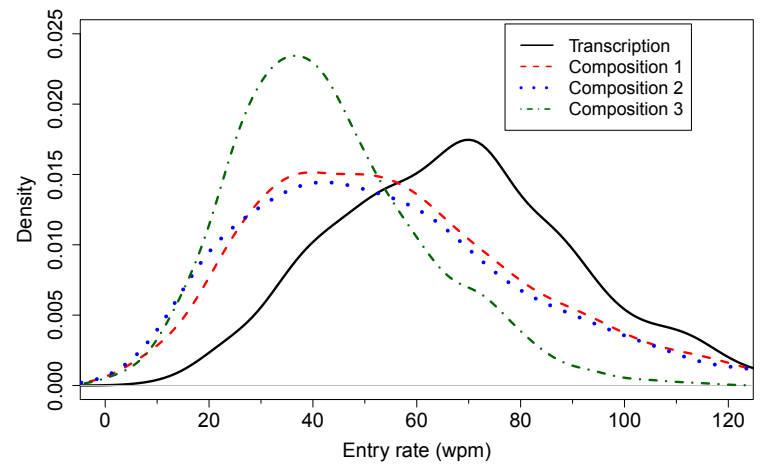

Figure 2. Probability densities for the transcription task and the three different composition tasks.

\section{DISCUSSION}

In theory, the inviscid entry rate estimate enables us to assess to what extent a mobile text entry methods hinders users' free-flowing writing. Such an analysis can tell us how far we are from the goal of achieving mobile text entry methods that support the fluid transmission of thoughts into writing. This analysis can in principle be carried out for various text entry methods by, for example, measuring participants' entry rates when they compose novel small messages.

Unfortunately, composition tasks are rarely used in text entry experiments. Instead the dominating method for testing a text entry method is to measure its performance using a transcription task. However, we can make an upper-bound estimate of a mobile text entry method's performance by basing a comparison on its highest reported transcription entry rate. If such an upper-bound estimate does not surpass our 67 wpm estimation of the sufficiently inviscid entry rate then the probability that the text entry method will ever support users' free-flowing writing is low.

We surveyed the literature to find the highest reported entry rates for an array of mobile text entry methods. The results are in Table 1. Note that these entry rates were obtained on transcription tasks rather than composition tasks and thus overestimate the entry rates of the methods in the table. Even so, Table 1 shows that very few text entry methods have reported entry rates near our estimate of the inviscid entry rate. This means there is still room for active research on faster mobile text entry methods.

\section{LIMITATIONS AND FUTURE WORK}

We defined the sufficiently inviscid entry rate for mobile text entry to be a population estimate of the mean composition entry rate plus one standard deviation. This definition is to some extent arbitrary. However, our definition has two advantages. First, it has a clear statistical interpretation. Second, even though it could be set higher, no existing mobile text entry method is close to the sufficiently inviscid entry rate.

It is likely there are alternative text entry methods that are already invented or yet to be discovered that could provide a better estimate of the true inviscid entry rate. We chose to measure the sufficiently inviscid entry rate based on the QWERTY keyboard for two reasons. First, a large proportion of the population are highly proficient with the QWERTY key- 
board. There is no easily accessible large population that have mastered a faster text entry method (such as stenography). Second, besides the QWERTY keyboard, the only other viable fast text entry method is speech. However, speech and writing processes generate significantly different texts [2]. Therefore, in order to estimate the inviscid entry rate based on speech it would be necessary to demonstrate that texts generated by speech are of as high quality (with high idea density, diverse vocabulary, lack of repetition or filler words, etc.) as text generated by other writing methods. This is an interesting avenue for future research.

Previously we defined the time it takes to a compose text as the sum of two variables: the time it takes to conceive the text and the time it takes to articulate the text. However, these two variables may not be independent. For speech, the cognitive resources required to conceive the text will in part be consumed by the additional cognitive resources required to articulate it [17]. Further work is necessary to tease out the relationship between these two variables.

\section{CONCLUSIONS}

It is obvious higher entry rates are better, but it is not as obvious when to stop searching for ever higher entry rates and start improving alternative design dimensions [10]. We suggest using the inviscid entry rate as an explicit goal that bounds the otherwise endless search for ever higher text entry rates. We have estimated the sufficiently inviscid text entry rate for mobile text entry to around $67 \mathrm{wpm}$. We compared existing mobile text entry methods against this estimate and found that the vast majority are substantially slower. Our analysis suggests the mobile text entry field needs to focus on methods that can viably approach the inviscid entry rate.

\section{REFERENCES}

1. Castellucci, S. J., and MacKenzie, I. S. Graffiti vs. Unistrokes: an empirical comparison. In Proc. $\mathrm{CHI}$ (2008), 305-308.

2. Chafe, W., and Tannen, D. The relation between written and spoken language. Annual Review of Anthropology 16 (1987), 383-407.

3. Clarkson, E., Clawson, J., Lyons, K., and Starner, T. An empirical study of typing rates on mini-QWERTY keyboards. In Ext. Abstracts CHI 2005 (2005), 1288-1291.

4. David, P. A. Clio and the economics of QWERTY. American Economic Review 75, 2 (1985), 332-337.

5. Goel, M., Findlater, L., and Wobbrock, J. WalkType: using accelerometer data to accomodate situational impairments in mobile touch screen text entry. In Proc. CHI (2012), 2687-2696.

6. Goel, M., Jansen, A., Mandel, T., Patel, S. N., and Wobbrock, J. O. ContextType: Using hand posture information to improve mobile touch screen text entry. In Proc. CHI 2013 (2013), 2795-2798.

7. James, C., and Reischel, K. Text input for mobile devices: comparing model prediction to actual performance. In Proc. CHI 2001 (2001), 365-371.
8. Kristensson, P., and Denby, L. C. Text entry performance of state of the art unconstrained handwriting recognition: a longitudinal user study. In Proc. CHI 2009 (2009), 567-570.

9. Kristensson, P. O. Discrete and Continuous Shape Writing for Text Entry and Control. Doctoral dissertation, Linköping University, 2007.

10. Kristensson, P. O. Five challenges for intelligent text entry methods. AI Magazine 30, 4 (2009), 85-94.

11. Lyons, K., Starner, T., and Gane, B. Experimental evaluations of the twiddler one-handed chording mobile keyboard. Human-Computer Interaction 21, 4 (2006), 343-392.

12. MacKenzie, I. S., and Zhang, S. X. The design and evaluation of a high-performance soft keyboard. In Proc. CHI 1999 (1999), 25-31.

13. Matias, E., MacKenzie, I., and Buxton, W. One-handed touch-typing on a qwerty keyboard. Human-Computer Interaction 11, 1 (1996), 1-27.

14. Oulasvirta, A., Reichel, A., Li, W., Zhang, Y., Bachynskyi, M., Vertanen, K., and Kristensson, P. O. Improving two-thumb text entry on touchscreen devices. In Proc. CHI (2013), 2765-2774.

15. Perlin, K. Quikwriting: continuous stylus-based text entry. In Proc. UIST (1998), 215-216.

16. Rosenbaum, D. Human Motor Control. Academic Press, 1991.

17. Shneiderman, B. The limits of speech recognition. Communications of the ACM 43, 9 (2000), 63-65.

18. Vertanen, K., and Kristensson, P. O. Parakeet: A continuous speech recognition system for mobile touch-screen devices. In Proc. IUI (2009), 237-246.

19. Vertanen, K., and Kristensson, P. O. A versatile dataset for text entry evaluations based on genuine mobile emails. In Proc. MobileHCI (2011), 295-298.

20. Vertanen, K., and Kristensson, P. O. Complementing text entry evaluations with a composition task. $A C M$ Transactions on Computer-Human Interaction 21, 2 (2014), Article No. 8.

21. Ward, D. J., Blackwell, A. F., and MacKay, D. J. C. Dasher: a gesture-driven data entry interface for mobile computing. Human-Computer Interaction 17, 2-3 (2002), 199-228.

22. Wigdor, D., and Balakrishnan, R. TiltText: using tilt for text input to mobile phones. In Proc. UIST (2003), $81-90$.

23. Wigdor, D., and Balakrishnan, R. A comparison of consecutive and concurrent input text entry techniques for mobile phones. In Proc. CHI (2004), 81-88.

24. Wobbrock, J. O., Myers, B. A., and Kembel, J. A. EdgeWrite: a stylus-based text entry method designed for high accuracy and stability of motion. In Proc. UIST (2003), 61-70. 